8-1-2020

\title{
Relative size underlies alternative morph development in a salamander
}

\author{
Michael P. Moore \\ Murray State University, moore.evo.eco@gmail.com \\ Joseph H. K. Pechmann \\ Western Carolina University \\ Howard H. Whiteman \\ Murray State University, hwhiteman@murraystate.edu
}

Follow this and additional works at: https://digitalcommons.murraystate.edu/faculty

Part of the Biology Commons

\section{Recommended Citation}

Moore, M.P., Pechmann, J.H.K. \& Whiteman, H.H. Relative size underlies alternative morph development in a salamander. Oecologia (2020). https://doi.org/10.1007/s00442-020-04723-8

This Peer Reviewed/Refereed Publication is brought to you for free and open access by Murray State's Digital Commons. It has been accepted for inclusion in Faculty \& Staff Research and Creative Activity by an authorized administrator of Murray State's Digital Commons. For more information, please contact msu.digitalcommons@murraystate.edu. 
1 TITLE: Relative size underlies alternative morph development in a salamander

2

3 AUTHORS: Michael P. Moore ${ }^{1,2,5}$, Joseph H.K. Pechmann ${ }^{3,4}$, and Howard H. Whiteman ${ }^{1,4}$

4

5 1. Watershed Studies Institute and Department of Biological Sciences, Murray State University,

6 Murray, KY 42071

7 2. Department of Biology, Case Western Reserve University, Cleveland, OH 44106.

8 3. Department of Biology, Western Carolina University, Cullowhee, NC 28723

9 4. Savannah River Ecology Laboratory, University of Georgia, Aiken, SC 29802

10 5. Present Address: Living Earth Collaborative, Washington University, St. Louis, MO 63105.

11 Email: moore.evo.eco@gmail.com

12

13 RUNNING TITLE: Relative size and polyphenism

14

15 AUTHOR CONTRIBUTIONS: HHW and JHK designed the study and collected all data.

16 MPM conducted all analyses and wrote the manuscript. All authors contributed to subsequent

17 revisions.

18

19

20

21

22 


\section{ABSTRACT}

24 Size thresholds commonly underlie the induction of alternative morphological states. However,

25 the respective importance of absolute and relative size to such thresholds remains uncertain. If

26 absolute size governs expression, morph frequency should differ among environments that

27 influence absolute sizes (e.g. resources, competition), and individuals of the same morph should

28 have similar average sizes across environments. If relative size determines expression, the

29 frequency of each morph may not differ among environments, but morphs within each

30 environment should differ in size relative to one another. We tested these predictions in a

31 salamander (Ambystoma talpoideum) that develops into either a terrestrial metamorph or an

32 aquatic paedomorph. To generate size variation within and among environments, we reared

33 individuals in mesocosm ponds across three conspecific densities. We found that morph

34 frequency did not differ among density treatments, and the morphs were not similarly sized

35 within each density treatment. Instead, within each environment, relatively larger individuals

36 became metamorphs and relatively smaller individuals became paedomorphs. Relative size

37 therefore determined morph development, highlighting the importance of an individual's social

38 context to size-dependent morph induction.

40 Keywords: facultative paedomorphosis; intraspecific competition; life-history variation;

41 polyphenism; resource polymorphism 


\section{INTRODUCTION}

45 Discrete, alternative phenotypes are taxonomically widespread and often enable individuals to 46 optimize fitness through differential resource use (Smith and Skúlason 1996). The induction of

47 these morphs commonly depends on a suite of external and internal factors, whereby even 48 genetically identical individuals can express alternative phenotypes based on differing

49 developmental environments (Nijhout 2003; West-Eberhard 2003). Such "polyphenisms"

50 broaden the range of environmental and social circumstances to which a single genotype can

51 produce well-adapted phenotypes (Sultan and Spencer 2002; Nijhout 2003). Polyphenisms also

52 appear to regulate the diversification patterns of many lineages (Pfennig et al. 2010). Identifying

53 the factors underlying polyphenic expression, as well as the ways in which they influence

54 development, therefore can provide insight into the origins and maintenance of alternative

55 phenotypes (Smith and Skúlason 1996; Moczek et al. 2011) and of phenotypic diversity more

56 generally (West-Eberhard 2003; Pfennig et al. 2010).

57 One factor that is commonly associated with alternative morph development is body size

58 (e.g. Wheeler 1991; Tomkins et al. 2011; Phillis et al. 2016), likely because the advantages of

59 each morph usually also depend on an individual's size (e.g. Michalczyk et al. 2018). Size-

60 dependent morph development can arise via the non-mutually exclusive effects of two features

61 of an individual's size: its absolute size and its relative size. When a population's size

62 distribution is temporally constant, morph development should evolve to depend entirely on an

63 individual's absolute size (Tachiki and Koizumi 2016). Under such conditions, morph induction

64 typically occurs if an individual surpasses energetic thresholds that are themselves correlated

65 with absolute size (Nijhout 2003; Tomkins and Moczek 2009). Alternatively, when a

66 population's size distribution varies across generations, and negative frequency-dependent 
67 selection maintains both morphs in the population, morph development should evolve to rely on

68 an individual's size relative to its competitors (i.e. relative size; Tachiki and Koizumi 2016).

69 Here, the relatively largest individuals express one morph, while the relatively smallest express

70 the other (Tompkins and Hazel 2007). In these cases, relative size often determines the outcome

71 of competition for essential resources (e.g. Ziemba and Collins 1999), which links it to the

72 physiological factors that underlie morph induction (e.g. Lorenzi et al. 2012). Although the

73 effects of absolute and relative size on the expression of behavioral alternatives are well

74 characterized (e.g. alternative mating strategies; Gross 1996; Tomkins and Hazel 2007), their

75 effects on the expression of life-history and/or morphological alternatives remain less well

76 studied (Maret and Collins 1994; Warner et al. 1996; Frankino and Pfennig 2001).

77 Facultative paedomorphosis in salamanders - the development of a reproductive aquatic

78 "paedomorphic" phenotype instead of, or sometimes prior to, a terrestrial "metamorphic"

79 phenotype - is well suited for assessing how absolute and relative size influence morph

80 development. Theory extended from the classic models of amphibian metamorphosis (Wilbur

81 and Collins 1973; Werner 1986; Rowe and Ludwig 1991) predicts two size-dependent

82 trajectories of induction (Whiteman 1994). When aquatic conditions promote high survival and

83 rapid growth (e.g. high resources, low predation), the largest individuals within these

84 environments should become paedomorphs while smaller individuals metamorphose to escape

85 competition from their larger counterparts ("Paedomorph Advantage" mechanism). In contrast,

86 when aquatic conditions are less favorable to survival and growth (e.g. low resources, high

87 predation), the largest individuals should escape from the natal habitat via metamorphosis

88 whereas individuals that do not reach minimum thresholds for metamorphosis mature as

89 paedomorphs rather than waiting to attain larger sizes ("Best-of-a-Bad Lot" mechanism). While 
90 morphs arising across natural and experimentally manipulated environmental gradients often

91 differ in size (reviewed in Whiteman 1994; Denoël et al. 2005), the respective importance of

92 absolute and relative size to induction is unknown.

93 We evaluated the influence of absolute and relative size on morph development in the

94 polyphenic salamander Ambystoma talpoideium. We began by raising larvae in mesocosm ponds

95 across three conspecific densities. We then used the resulting size variation within and across

96 environments to assess how relative and absolute size influence morph development. If relative

97 size affects morph expression, morph frequencies may be similar among density treatments, and

98 one morph will be consistently larger than the other within each mesocosm pond and within each

99 density treatment. If absolute size influences morph development, morph frequencies will differ

100 among density treatments that influence absolute sizes, and the average size of each morph

101 should be similar regardless of the mesocosm pond or density treatment. Finally, the effects of

102 relative size could also depend on absolute size, if, for instance, relative size becomes important

103 only after some minimum absolute size is reached. In such cases, one morph may: 1) only occur

104 above some minimum absolute size; 2) be very rare in high-density treatments, where all

105 individuals are small, but be equally as common as the other morph in medium- and low-density

106 treatments, where individuals are larger; and 3) be consistently larger than the other morph

107 within the subset of tanks and density treatments in which they are both induced.

108

109 METHODS

110 Study System and Collection

111 Ambystoma talpoideum is found in the southeastern United States and northward along the

112 Mississippi River Valley into southern Illinois (Petranka 1998). Individuals typically mature as 
113 paedomorphs or undergo metamorphosis before their first winter or prior to maturing in the

114 following spring (Petranka 1998). Paedomorphic A. talpoideum remain as mature adults in the

115 aquatic environment or will sometimes subsequently undergo metamorphosis (e.g. Doyle and

116 Whiteman 2008); however, metamorphs cannot become paedomorphs. Relative to other nearby

117 populations, our source population (Ellenton Bay, Savannah River Site, SC) has a longer larval

118 period, higher frequency of paedomorphosis, and greater sensitivity to pond drying (Semlitsch et 119 al. 1990).

120 To generate individuals for this experiment, we bred adults by releasing 9 males and 15

121 females into two $1000 \mathrm{~L}$ polyurethane tanks filled with aged water and leaf litter at the Savannah

122 River Ecological Laboratory (SREL). From these tanks, we collected 1008 embryos, which we

123 then mixed and randomly assigned by treatment (see below) into our experimental ponds.

\section{Experimental Design}

126 We reared larvae in $1000 \mathrm{~L}$ polyurethane tanks at one of three densities $($ Low $=10$, Medium $=$

12724 , High $=50$ individuals per tank; $\mathrm{n}=12$ tanks per density), chosen to mimic the range of

128 natural competition and to generate relatively continuous size variation (Semlitsch 1987). After

129 filling the tanks with well water and $1.75 \mathrm{~kg}$ of leaf litter, we covered them with mesh lids to

130 prevent colonization by Hylid treefrogs and predatory macroinvertebrates (e.g. Aeshnidae and

131 Dytiscidae spp.). Standpipes maintained each tank's water depth at $45 \mathrm{~cm}$. Starting at the

132 beginning of the experiment, we also periodically added $1.5 \mathrm{~L}$ of concentrated, field-collected

133 plankton to each tank, providing a food source for the larvae. A mesocosm approach was well

134 suited for our study because the focal species can complete its entire life cycle in ponds of this

135 size, and these venues possess features of natural ponds that cannot easily be simulated in lab 
136 studies (e.g. self-sustaining food web, natural photoperiod, diurnal temperature changes; Wilbur 137 1997; Semlitsch and Boone 2009).

138 We introduced the salamanders to tanks on 10 March 1997 (Day 1). To gain a fuller

139 picture of morph expression (see also Doyle and Whiteman 2008), the study concluded the

140 following May (after 438 days). In the final week, half of the tanks were accidentally dried much

141 faster than is natural (i.e. overnight). As this drying did not affect the probability of expressing

142 one morph or the other (all $P>0.223$ ), or the relationships between morph expression and body

143 size (all $P>0.253$ ), it does not bias our results and conclusions (see Electronic Supplementary

144 Materials, ESM; Table S1).

145 We captured individuals with minnow traps on days 76-77 (May 1997), 226-228

146 (October 1997), 297-303 (January 1998), 363-366 (March 1998), and 437-438 (May 1998). We

147 also set out and regularly checked floating plastic boxes with mesh ramps, which allowed

148 metamorphs to climb out of the water. We measured each captured individual's snout-vent

149 length (SVL) to the nearest $0.5 \mathrm{~mm}$ using a ruler and then returned all gilled individuals to tanks.

150 We focused on SVL, rather than mass, because it changes less across metamorphosis and is not 151 confounded with egg production by paedomorphic females (Dodd 2010). Once the larvae were

152 large enough to handle without injuring them (day 226), we uniquely toe clipped each captured

153 individual with microdissecting scissors. Individuals were considered metamorphs if they had 154 resorbed their gills and tail fin, and we removed them from their tanks upon observation. To 155 avoid destructive sampling and decreasing tank densities, we differentiated paedomorphs from 156 larvae prior to the final sample by visually inspecting their cloacae for swelling and pigmentation 157 and by "candling" with a fiber optic light to observe eggs. On day 438, we emptied all tanks and 158 recovered and measured all remaining survivors. We sacrificed animals with MS-222, fixed them 
159 in $10 \%$ formalin, and preserved them in $70 \%$ ethanol. We dissected aquatic individuals to

160 differentiate paedomorphs from larvae. Paedomorphic males have enlarged, pigmented vasa

161 deferentia and testes, while paedomorphic females have pigmented ova (Semlitsch 1987).

162

163 STATISTICAL ANALYSES

164 We conducted analyses with R v.3.6.0 (R Core Team 2019). For analyses of morph and size, we 165 used an individual's traits at the final capture except where noted (see below for further 166 rationale). Mixed-effects models were fit using 'Ime4' (Bates et al. 2015). When used,

167 significance tests were conducted with likelihood ratio tests in which we compared a model with 168 all of the effects to one without the effect of interest. Post-hoc tests were conducted using the 169 lsmeans package (Lenth 2016). We report parameter estimates \pm SE from the fitted models. 170 We began by comparing survival among density treatments using a generalized linear 171 model with a quasibinomial error distribution. We also verified the presence of size differences 172 among density treatments using a linear mixed-effects model with each individual's SVL as the 173 response, density as a fixed effect, and tank as a random effect.

174 To first determine if morphs differed in average body size across the entire study (e.g.

175 Semlitsch 1987; Doyle \& Whiteman 2008), we assessed overall size differences between

176 metamorphs and paedomorphs using a linear mixed-effects model with each individual's final

177 SVL as the response, its morph as a fixed effect, and its tank as a random intercept. Next,

178 because our study design did not experimentally manipulate each individual's position in the size 179 hierarchy within its tank, and there was not a single best analysis to conduct, we used several 180 approaches to examine the effects of absolute and relative size on morph induction. 
If morph expression depends on absolute size variation, then the frequency of each morph

182 within a tank should be related to the average size of individuals in that tank. We used two

183 analyses to test this. We first used a generalized linear model with a quasibinomial error

184 distribution to examine if the proportion of individuals expressing each morph (arbitrarily scored

185 as $1=$ metamorph, $0=$ paedomorph) within a tank varied with the average size of the individuals

186 within that tank. We also used a similar model to test for differences in morph expression among

187 density treatments, which explained the vast majority of variation in tank-mean size $\left(\mathrm{R}^{2}=0.900\right.$;

$\left.188 \mathrm{~F}_{2,33}=149.0, P<0.001\right)$. Because of their strong association, and because we were interested in the

189 overall effect of tank-mean size and of density treatment, rather than the effect of either one after

190 controlling for the other, we report the results from separate models. However, results were

191 qualitatively similar even when fitting a model with both terms simultaneously.

192 Next, for individuals within those tanks where both morphs developed, we used a multi-

193 model inference approach to examine the effects of absolute and relative size on morph

194 expression. First, we fit a generalized linear mixed-effects model that included an individual's

195 morph as the response (scored as above); its relative size, absolute size, and their interaction as

196 fixed effects; and tank as a random effect. Here, relative size was calculated as the mean SVL of

197 an individual's tankmates subtracted from the individual's SVL. To improve model convergence,

198 we also "centered" an individual's absolute size by subtracting the mean SVL of all individuals

199 in the experiment from the individual's SVL (Schielzeth 2010). Using AIC , which is less

200 sensitive to issues of correlated predictors than likelihood ratio tests (Freckleton et al. 2011;

201 Grueber et al. 2011), we compared models with all possible combinations of relative and

202 absolute size via the 'dredge' function in MuMIn (Bartoń 2019). Models were considered

203 equivalent if they had the same number of parameters and were separated by less than 2 
204 (Burnham and Anderson 2002). Despite the relationship between relative size and absolute size,

205 simulations indicated that this multi-model inference approach rarely excludes an important term

206 from the best-supported model (see ESM for full details). To evaluate the magnitude and

207 direction of any effects that absolute and relative size have on morph expression, we used either

208 the parameter estimates of the best-supported model or model-averaged estimates for sets of

209 equally supported models $\left(\Delta \mathrm{AIC}_{\mathrm{c}}<2\right)$. We also compared another set of models beginning with

210 all of the terms described above, except an individual's density treatment was included instead of

211 its absolute size. If an individual's relative size controls morph expression, then relative size will

212 be included in the best-supported models. If an individual's absolute size determines morph

213 expression, then the best-supported model will include an individual's absolute size or its density

214 treatment, which controls the vast majority of variation in absolute size. If, for example, relative

215 size only affects morph expression after some minimum absolute size has been reached or only

216 in some densities, then the interaction between relative size and absolute size or between relative

217 size and density should be in the best-supported model. Because of the very strong association

218 between an individual's size and its tank's density treatment, a single global model

219 simultaneously including fixed effects of density, absolute size, and relative size did not

220 converge. However, we present several other analyses using this approach, including models of

221 absolute size and density, in the Electronic Supplementary Materials. In all cases, the results

222 from these analyses align with the those presented in the main text (ESM Table S3).

223 Given the variation in size and morph frequencies among tanks (see Results), we next

224 tested if one morph was consistently larger than tankmates of the other morph. For each tank

225 where both were produced, we first averaged the sizes of its metamorphs and paedomorphs.

226 Next, we counted the tanks where the average size of each morph was larger than that of the 
227 other, and then we used a $\chi^{2}$ test to evaluate if one morph was the larger of the two within each

228 tank more often than would be expected by chance. Importantly, if the body-size distribution

229 within every tank was similar, this approach would not clarify the role of relative size. However,

230 this was not the case (see Results), and one morph being consistently larger than the other within

231 each tank would support a role for relative size.

232 Finally, we compared the morphs' sizes using a linear mixed-effects model with density

233 and morph as fixed effects. Tank was fitted as a random effect, and metamorphs and

234 paedomorphs across all tanks were included. This approach compares the two morphs' sizes after

235 accounting for the variation in size associated with the individual's density treatment. It therefore

236 tests if morphs are different sizes within each density. As density explained the vast majority of

237 variation in tank-mean size, size differences between the morphs within each density should

238 emerge if there are effects of relative-size variation within the tanks. One additional benefit of

239 this approach is that it allowed us to test if the smaller morph in one density was larger than the

240 larger morph in a higher density — a pattern indicative of relative-size effects. If relative size

241 underlies morph development, then, after accounting for the effects of density, morph should be

242 associated with SVL. Moreover, if the effect of relative size on morph expression differs among

243 density treatments (Whiteman 1994), we could find a significant morph by density interaction,

244 whereby one morph is larger in low-density treatments but smaller in high-density treatments.

245 Although only one individual underwent metamorphosis prior to the final month of the

246 experiment (April 1998), following individuals across ontogeny would yield insight into how

247 absolute and relative size affect development. However, several supplemental analyses indicate

248 that using traits at the end of the experiment is unlikely to bias our conclusions (see ESM for full

249 details). First, among the small subset of marked individuals that were recovered and identified 
250 at the end of the experiment (63 out of 163 total metamorphs and paedomorphs, $38.7 \%$ ), those 251 that became paedomorphs and then metamorphosed did not differ in absolute $(\mathrm{t}=1.4, P=0.17)$ or

252 relative SVL ( $\mathrm{t}=0.9, P=0.38)$ from those that metamorphosed directly from the larval state

253 (ESM, Table S2). Similarly, among the smaller subset of individuals who were marked in

254 October 1997 and recaptured as metamorphs or paedomorphs, their size at this time was strongly 255 related to their size in May $1998\left(\mathrm{R}^{2}=0.755, \mathrm{~F}_{1,31}=95.5, P<0.001\right)$. Growth between these time 256 points also did not differ substantially among the developmental trajectories $\left(\chi_{2}^{2}=5.0, P=0.08\right)$.

257 Thus, final size reasonably reflects size when morph development typically begins.

\section{RESULTS}

260 Survival did not differ significantly among density treatments $\left(\mathrm{F}_{2,33}=2.7, P=0.08\right)$, but body size

$261 \operatorname{did}\left(\chi_{2}^{2}=53.8, P<0.001\right.$; all pairwise linear contrasts $\left.P<0.001\right)$. Additionally, across the study, 262 metamorphs were $3.29 \pm 0.61 \mathrm{~mm}$ larger than paedomorphs $\left(\chi_{1}^{2}=27.2, P<0.001\right.$; Fig. 1$)$.

263 The frequency of metamorphs and paedomorphs within each tank was not related to the

264 mean body size within the tank $\left(\chi_{1}^{2}=1.4, P=0.25\right)$ or to the tank's density treatment $\left(\chi_{2}^{2}=1.9\right.$, $265 P=0.39)$.

266 Among those individuals that were reared within the 15 tanks where both morphs

267 developed, we next used a multi-model inference approach to assess the effects of an

268 individual's absolute and relative size on morph expression. A model including only relative size

269 had the most support, though a more complex model that included both relative size and absolute

270 size had a similar, but slightly worse, $\mathrm{AIC}_{\mathrm{c}}$ score (Table 1). Model-averaged parameter estimates

271 indicated that, for a given absolute size, the log-odds of metamorphosis increased by $0.384 \pm$

2720.142 for every $1 \mathrm{~mm}$ increase in relative size (95\% CIs: 0.103-0.665; Fig 1A). In contrast, for a 
273 given relative size, the log-odds of metamorphosis only increased by $0.045 \pm 0.081$ for a $1 \mathrm{~mm}$

274 increase in absolute size (95\% CIs: -0.115-0.205). We also compared candidate models starting

275 from one that included an individual's relative size, its density treatment, and the interaction

276 between relative size and density treatment (Table 1). Among these candidate models, only the

277 model including relative size was well supported (Table 1), with the log-odds of an individual

278 undergoing metamorphosis increasing by $0.421 \pm 0.130$ for every $1 \mathrm{~mm}$ in relative size $(95 \%$

279 CIs: 0.196-0.719). Collectively, these two comparisons indicate that the relatively largest

280 individuals within tanks and within density treatments underwent metamorphosis. Alternative

281 approaches for examining the effects of absolute and relative size were also consistent with a

282 strong effect of relative size (ESM, Table S3). Likewise, a multi-model inference approach

283 revealed a strong effect of relative size when calculating relative size as an individual's size

284 divided by the mean size of its tankmates (ESM, Table S3).

285 We next assessed if one morph was usually the larger of the two within the tanks where

286 both developed (2 high-density tanks, 5 medium-density tanks, and 8 low-density tanks). Despite

287 the density treatment causing large differences in the average size of individuals among these

288 tanks, metamorphs were larger, on average, than their paedomorph tankmates within $93 \%$ of

$289 \operatorname{tanks}\left(\chi_{1}^{2}=11.3, P<0.001\right)$.

290 After accounting for overall density-dependent reductions in size $\left(\chi_{2}^{2}=63.2, P<0.001\right)$,

291 metamorphs were consistently larger than paedomorphs within each density (morph: $\chi_{1}^{2}=36.7$,

$292 P<0.001$; interaction: $\chi_{2}^{2}=4.1, P=0.13$, Fig. 1B). Notably, paedomorphs from low-density

293 treatments were larger than metamorphs from medium-density treatments (linear contrast: $\mathrm{t}=2.8$,

$294 P=0.008$; Fig. 1B), though medium-density paedomorphs were not different from high-density

295 metamorphs $(\mathrm{t}=1.4, P=0.54)$. Results were similar when focusing only on the tanks where both 
morphs developed (ESM, Table S4). Individuals that were marked and re-captured also showed

297 this pattern $\left(\chi_{2}^{2}=27.2, P<0.001\right)$. Within density treatments, paedomorphs were smaller than

298 metamorphs that transitioned directly from the larval state $(\mathrm{t}=-4.6, P<0.001)$ and metamorphs

299 that became paedomorphs first (t=-3.2, $P=0.006$; ESM, Fig. S1).

\section{DISCUSSION}

302 We evaluated the effects of relative and absolute size on morph development in a salamander,

303 Ambystoma talpoideum. Metamorphs tended to be larger than paedomorphs across the study, and

304 multiple lines of evidence indicate that relative size strongly influenced this pattern. First, several

305 multi-model inference approaches found that the relatively largest individuals within each tank

306 and within each density treatment were more likely to undergo metamorphosis while relatively

307 smaller individuals were more likely to become paedomorphs. Second, even though the size

308 distribution differed among tanks and density treatments, metamorphs averaged larger sizes than

309 paedomorphs within nearly all tanks. Third, metamorphs were also consistently larger than

310 paedomorphs within each density treatment. Finally, despite being smaller than metamorphs

311 from low-density treatments, paedomorphs from low-density treatments were larger than

312 metamorphs from medium- and high-density treatments. In contrast, we found little support for

313 an effect of absolute size on morph expression. First, the proportion of morphs within each tank

314 was not related to either the average size of individuals in the tank or to the tank's density

315 treatment. Second, although absolute size was included in a well-supported model that also

316 included relative size, this model had a worse $\mathrm{AIC}_{\mathrm{c}}$ score and was less parsimonious than the

317 best-supported model, which only included relative size. The effect of absolute size on morph

318 expression also had confidence intervals that overlapped 0 . Third, rather than individuals from 
319 the same morph being similarly sized across density treatments, morphs were consistently

320 different sizes within each density treatment. While manipulations that place absolutely large

321 individuals at the smaller end of a population size distribution, and vice versa, could empirically

322 disentangle the effects of absolute and relative size (sensu Maret and Collins 1994; Van Buskirk

323 et al. 2017), our correlative findings indicate that relative size regulates morph development.

324 Size-dependent models of morph development often assume absolute-size thresholds

325 (e.g. Wheeler 1991; Emlen and Nijhout 2000; Chapman et al. 2011). Among the size-dependent

326 models of facultative paedomorphosis, our results are largely consistent with the "Best-of-a-Bad

327 Lot" trajectory, whereby individuals become paedomorphs when they cannot reach the minimum

328 threshold for metamorphosis (Whiteman 1994). However, we did not find much evidence for

329 these absolute-size thresholds, nor did we find that the effect of relative size changed with the

330 favorability of the aquatic environment for growth (cf. Whiteman 1994). Other natural and

331 mesocosm studies that have considered competition have also found patterns of morph

332 expression and size differences that are consistent with the "Best-of-a-Bad Lot" trajectory (e.g.

333 Doyle and Whiteman 2008; Whiteman et al. 2012; Lackey et al. 2019), but this is the first to

334 show that relative size is largely responsible. Given these findings, it may be valuable to expand

335 size-dependent models of this polyphenism to include a larger role for relative size.

336 Because relative size influences morph expression, understanding the factors that

337 generate variation in relative size should be an emphasis for future research. Previous work has

338 shown that size variation often stems from many factors (Ziemba and Collins 1999), including

339 additive genetic effects on growth or resource acquisition (Mousseau and Roff 1987; Watkins

340 and McPeek 2006), maternal effects on hatching size or hatching date (Moore et al. 2015; Moore

341 et al. 2019), and fortuitous consumption of high-value resources early in life (Wilbur and Collins 
342 1973; Álvarez and Nicieza 2002; Whiteman et al. 2003). Once such variation arises, however,

343 interference competition will reinforce it: relatively larger individuals will subsequently

344 outcompete smaller individuals and maintain their status in the hierarchy (Ziemba and Collins

345 1999; Johnson et al. 2003; Buston 2003). Although we do not know what caused relative size to

346 vary in our study, the strong correlation between size in October 1997 and size in May 1998 is

347 consistent with variation arising early in life and being maintained via such reinforcement. In

348 light of its importance to morph expression, examining the sources of relative size variation in

349 natural settings could provide insight into the origins and maintenance of facultative

350 paedomorphosis and, perhaps, other polyphenisms (Whiteman et al. 2012).

351 Whereas relative size strongly affected morph expression in this study, we found little

352 evidence that absolute size was particularly impactful. However, absolute size could be more

353 influential under other conditions. Our study generated a wide range of body sizes but still might

354 not have created small enough average sizes to observe effects of absolute size on morph

355 frequency or to identify some minimum threshold for metamorphosis (Wilbur and Collins 1973).

356 Likewise, our low-density treatments were higher than in some studies (cf. Semlitsch 1987;

357 Anderson and Whiteman 2015) and were higher than the very lowest densities observed in

358 natural populations (Anderson et al. 2018). Although it seems unlikely that using even more

359 extreme densities would have changed the frequency of metamorphosis or importance of

360 absolute size, other studies have produced a higher frequency of metamorphs than our study (e.g.

361 Semlitsch 1987; Doyle and Whiteman 2008; Anderson and Whiteman 2015). We therefore

362 cannot rule out the possibility that using even more extreme density treatments would have

363 increased the importance of absolute size. Beyond intra-cohort competition, absolute size could

364 also be influential in the context of other environmental factors. For instance, in natural 
365

366

367

368

369

370

371

372

373

374

375

376

377

378

379

380

381

382

383

384

385

386

387

permanent or semi-permanent ponds, aquatic ambystomatids from previous cohorts compete

with and consume young-of-the-year (Wissinger et al. 2010; Whiteman et al. 2012; Anderson et

al. 2013). Size asymmetries of these inter-cohort interactions could render the aquatic

environment so unsuitable that only the very smallest individuals become paedomorphs

(Whiteman et al. 2012). Absolute-size thresholds could also differ among populations. In

organisms with environmental sex determination, for example, sex expression often occurs after achieving some fraction of an individual's maximum possible body size, but the exact threshold varies among populations and species (Munday et al. 2006). This subsequently alters the

likelihood of reaching the absolute-size threshold and, therefore, also whether or not relative size can play any role at all (e.g. Collin 2006). Thus, although our results show that relative size can determine morph expression over a range of ecologically relevant conditions, disentangling the effects of absolute and relative size across a wider array of experimental and natural environments remains necessary.

We tested here if morph expression depends on relative size, yet exploring the ecological and physiological consequences of relative size variation will inform why morph expression has evolved to rely on it in the first place. Relative size often affects an individual's competitive outcomes more than absolute size (e.g. Warner et al. 1996; Ziemba and Collins 1999). Provided that relative size remains reliably linked to competitive outcomes, this has at least two consequences. First, because relative size determines the outcome of interference competition (Ziemba and Collins 1999), relatively larger individuals could be better at acquiring resources necessary for morph induction (Frankino and Pfennig 2001; Johnson et al. 2003). In this way, relative size controls induction via acquisition differences of essential resources. However, under such a scenario, absolute size seems like it should also have strong effects, which it did not in 
388 this study. Alternatively, because of that same relationship between relative size and competitive

389 outcomes, individuals of the same relative size may experience their respective environments

390 similarly, and as a result, have similar physiological responses (Lorenzi et al. 2012). While the

391 neural and hormonal underpinnings of facultative paedomorphosis are poorly understood,

392 previous work has implicated the thyroxine hormone cascade (Voss et al. 2012), which interacts

393 with stress hormone concentrations (Denver 2009). These pathways require more research, and

394 experiments that manipulate circulating hormones and relate them to morph expression in

395 mesocosm and natural settings could illuminate the proximate mechanisms driving morph

396 expression.

397 As with the proximate factors linking relative size to morph expression, exploring the

398 selective pressures that have favored this relationship will provide insight into the evolution of

399 resource polyphenisms. For any cue to adaptively induce a resource polyphenism, it must predict

400 the future competitive outcomes for that individual (Nijhout 2003). Small relative size, and its

401 associated eco-physiological factors, may be a reliable cue if individuals retain their position in

402 the size hierarchy across life stages. Such a pattern is likely in A. talpoideum because: 1) growth

403 between metamorphosis and maturity is small (Semlitsch et al. 1988); 2) metamorphs typically

404 do not emigrate far into the terrestrial environment (Scott et al. 2013); and 3) paedomorphs

405 remain in their natal ponds. Additionally, for morph induction to be adaptively cued by small

406 relative size in any organism, those individuals must have higher fitness as one morph than they

407 would have had as the other (Roff 1996; West-Eberhard 2003). Paedomorphosis could be

408 favored for these individuals if relatively small metamorphs are outcompeted for the best

409 burrows and foraging opportunities by larger terrestrial counterparts (Searcy et al. 2014). With

410 larger individuals leaving the pond, reduced competition may also offset other unfavorable 
411 conditions for relatively small individuals that remain in the pond as paedomorphs. Earlier

412 maturity and/or increased breeding frequency of paedomorphs (Whiteman 1997; Ryan and

413 Plague 2004; Lackey et al. 2019) could further compensate for any costs of being relatively

414 smaller than metamorphs during reproduction (e.g. mate attraction, Whiteman et al. 2006;

415 relative fecundity, Lackey et al. 2019). Because intense interactions with competitors frequently

416 persist across the life cycle, being relatively smaller than competitors in an earlier stage may

417 often predict lifetime competitive outcomes and, therefore, could be a cue for many resource

418 polyphenisms (Pfennig and Pfennig 2009).

419 It is also intriguing why selection would favor metamorphosis for relatively large

420 individuals that had likely been performing successfully in the aquatic habitat (Asquith and

421 Vonesh 2013; Michalczyk et al. 2018). One plausible explanation is that metamorphosis is

422 simply the default trajectory, and being relatively large provides no new information to shift

423 individuals from this path. Paedomorphosis, not metamorphosis, would then be sensitive to

424 relative size, which would mirror situations where the default morph is developed unless some

425 external factor induces the alternative (e.g. heterophyllic leaves, Cook 1968; carnivore-morph

426 tadpoles, Pfennig 1990). Being relatively large could also provide less reliable information than,

427 and be overridden by, extrinsic cues that indicated metamorphosis was their best option overall

428 (Whiteman 1994; Denoël et al. 2005). For example, under high desiccation or predation risk, the 429 cues associated with being larger than one's competitors may not be very predictive of one's

430 fitness prospects and could be ignored in favor of more informative cues (Werner 1986; Rowe

431 and Ludwig 1991). The fitness benefits of metamorphosis for relatively large individuals would

432 be clarified by experimentally inducing the expression of each morph in individuals across the 
433 full range of relative sizes and then measuring their lifetime reproductive success (sensu Warner 434 and Shine 2008).

435 Overall, our results indicate that relative size underlies facultative paedomorphosis in

436 salamanders. Given the historical emphasis on absolute size, it would be useful to expand some

437 of the models of size-dependent morph expression to include a greater role for relative size.

438 Furthermore, habitat differences may favor varying degrees of reliance on relative size. The

439 specific thresholds for induction could therefore differ greatly, as has occurred in some

440 alternative mating tactics (Gerhardt et al. 1987) and environmental sex determination (Warner et

441 al. 1996). For instance, relative size as an aquatic larva may predict competitive outcomes in

442 later terrestrial stages less reliably in populations sourced by multiple natal ponds (Moore and

443 Whiteman 2016; Lackey et al. 2019). In such cases, selection could reduce reliance on relative

444 size and/or could increase the relative-size threshold such that the only individuals that

445 metamorphose are those that are certain to compete successfully in the terrestrial environment

446 (Tachiki and Koizumi 2016). Many other taxa with resource polymorphisms have life cycles

447 where the environment of just one stage varies widely among populations (e.g Moczek and

448 Nijhout 2003; Tomkins and Brown 2004), and such stage-dependent environmental variation

449 could commonly promote diversification in how individuals integrate the information conveyed

450 by relative size during development (Tomkins et al. 2011; Moore and Martin 2019). Thus,

451 beyond identifying how frequently relative size influences morph expression, insight into the

452 origins and maintenance of many size-dependent morphs will emerge from investigating how

453 ecological factors shape the strength of this reliance.

455 ACKNOWLEDGEMENTS 
456 We thank the many mentors who have patiently awaited this work, especially J.W. Gibbons. T.

457 Ryan provided assistance in experimental monitoring. Feedback from T. Anderson, A. Lackey,

458 M. Dugas, and members of the CWRU Ecology and Evolution reading group improved the

459 presentation of the study. At various times throughout this long-gestating project, funding for

460 MPM was provided by the Watershed Studies Institute (WSI), an MSU Graduate Innovation

461 Assistantship, a GAANN Fellowship, the CWRU Department of Biology, as well as G.

462 Kornblum and the Living Earth Collaborative. JHKP was funded by the Savannah River Ecology

463 Laboratory (SREL), Florida International University, and Western Carolina University. HHW

464 was funded by the SREL, WSI, the MSU Department of Biological Sciences, an MSU CISR

465 award, and the NSF (DEB-1354787). Financial assistance was also provided by the Department

466 of Energy Office of Environmental Management under award number DE-EM0004391. This

467 report was prepared as an account of work sponsored by an agency of the United States

468 Government. Neither the United States Government nor any agency thereof, nor any of their

469 employees, makes any warranty, express or implied, or assumes any legal liability or

470 responsibility for the accuracy, completeness, or usefulness of any information, apparatus,

471 product, or process disclosed or represents that its use would not infringe privately owned rights.

472 Reference herein to any specific commercial product process, or service by trade name,

473 trademark, manufacturer, or otherwise does not necessarily constitute or imply its endorsement,

474 recommendation, or favoring by the United States Government or any agency thereof. The views

475 and opinions of authors expressed herein do not necessarily state or reflect those of the United

476 States Government or any agency thereof.

477

478 ETHICAL APPROVAL 
479 All applicable institutional and/or national guidelines for the care and use of animals were

480 followed.

481

\section{DATA AVAILABILITY}

483 Data used in these analyses available at Dryad Digital Repository

484 (doi:10.5061/dryad.sf7m0cg3p)

485

486

487

488

\section{REFERENCES}

Álvarez D, Nicieza AG (2002) Effects of temperature and food quality on anuran larval growth

$490 \quad$ larval salamander size and density. Copeia 105: 237-248.

491 Anderson TL, Mott CL, Levine TD, Whiteman HH (2013) Life cycle complexity influences

492 intraguild predation and cannibalism in pond communities. Copeia 2013: 284-291

493 Anderson TL, Whiteman HH (2015) Asymmetric effects of intra- and inter-specific competition

494 on a pond-breeding salamander. Ecology 96: 1681-1690

495 Asquith CM, Vonesh JR (2010) Effects of size and size structure on predation and inter-cohort

496 competition in red-eyed treefrog tadpoles. Oecologia 170: 629-639

497 Bartoń K (2019) MuMIn: Multi-model inference. R package version 1.43.6.

498 Bates D, Maechler M, Bolker B, Walker S (2015) lme4: Linear mixed-effects models using

$499 \quad$ lme4. J Stat Softw 67: 148

500 Burnham KP, Anderson DR (2002) Model selection and multimodel inference: a practical

501 information-theoretic approach. Springer, New York 
502 Buston PM (2003) Size and growth modification in clownfish. Nature 424: 145-146

503 Chapman BB, Brönmark C, Nilsson J-Å, Hansson L-A (2011) The ecology and evolution of 504 partial migration. Oikos 120: 1764-1775

505 Collin R (2006) Sex ratio, life-history invariants, and patterns of sex change in a family of 506 protandrous gastropods. Evolution 60: 735-745

507 Cook CDK (1968) Phenotypic plasticity with particular reference to three amphibious plant 508 species. In: Heywood V (ed) Modern methods in plant taxonomy. Academic Press, London, $509 \quad$ pp $97-111$

510 Denoël M, Joly P, Whiteman HH (2005) Evolutionary ecology of facultative paedomorphosis in 511 newts and salamanders. Biol Rev 80: 663-671

512 Denver RJ. 2009. Stress hormones mediate environment-genotype interactions during amphibian 513 development. Gen Comp Endocr 164: 20-31

514 Dodd CK (2010) Amphibian ecology and conservation: a handbook of techniques. Oxford $515 \quad$ University Press, Oxford

516 Doyle JM, Whiteman HH (2008) Paedomorphosis in Ambystoma talpoideum: effects of initial 517 body size variation and density. Oecologia 156: 87-94

518 Emlen DJ, Nijhout HF (2000) The development and evolution of exaggerated morphologies in 519 insects. Annu Rev Entomol 45: 661-708

520 Frankino WA, Pfennig DW (2001) Condition-dependent expression of trophic polyphenism:

521 effects of individual size and competitive ability. Evol Ecol Res 3: 939-951

522 Freckleton RP (2011) Dealing with collinearity in behavioral and ecological data: model

523 averaging and the problems of measurement error. Behav Ecol Sociobiol 65: 91-101 
524 Gerhardt HC, Daniel RE, Perrill SA, Schramm S (1987) Mating behaviour and male mating

$525 \quad$ success in the green treefrog. Anim Behav 35: 1490-1503

526 Gross MR (1996) Alternative reproductive strategies and tactics: diversity within sexes. Trends

$527 \quad$ Ecol Evol 11: 2-98

528 Grueber CE, Nakagawa S, Laws RJ, Jamieson IG (2011) Multimodel inference in ecology and 529 evolution: challenges and solutions. J Evol Biol 24: 699-711

530 Johnson EB, Beirzychudek P, Whiteman HH (2003) Potential of prey size and type to affect

531 foraging asymmetries in tiger salamander (Ambystoma tigrinum nebulosum) larvae. Can J

532 Zool 81: 1726-1735

533 Lackey ACR, Moore MP, Doyle J, Gerlanc N, Hagan A, Geile M, Eden C, Whiteman HH (2019)

534 Lifetime fitness, sex-specific life history, and the maintenance of a polyphenism. Am Nat 194:

$535 \quad 230-245$

536 Lenth RV (2016) Least-squares means: The R Package lsmeans. J Stat Soft 69: 1-33.

537 Lorenzi V, Earley RL, Grober MS (2012) Differential responses of brain, gonad and muscle

538 steroid levels to changes in social status and sex in a sequential and bidirectional

539 hermaphroditic fish. PLoS One 7: e51158

540 Maret TJ, Collins JP (1994) Individual responses to population size structure: the role of size

541 expression of a trophic polyphenism. Oecologia 100: 279-285

542 Michalczyk Ł, Dudziak M, Radwan J, Tomkins JL (2018) Fitness consequences of threshold trait

543 expression subject to environmental cues. Proc R Soc B 285: 20180783

544 Moczek AP, Sultan S, Foster S, Ledón-Rettig C, Dworkin I, Nijhout HF, et al. (2011) The role of

545 developmental plasticity in evolutionary innovation. Proc R Soc B 278: 2705-2713

546 Moczek AP, Nijhout HF (2003) Rapid evolution of polyphenic threshold. Evol Dev 5: 259-268 
547 Moore MP, Landberg T, Whiteman HH (2015) Maternal investment mediates offspring life

548 history variation with context-dependent fitness consequences. Ecology 96: 2499-2509

549 Moore MP, Martin RA (2019) On the evolution of carry-over effects. J Anim Ecol 88: 1832-

$550 \quad 1844$

551 Moore MP, Whiteman HH (2016) Natal philopatry varies with larval condition in salamanders.

552 Behav Ecol Sociobiol 70: 1247-1255

553 Moore MP, Whiteman HH, Martin RA (2019) A mother's legacy: the strength of maternal

554 effects in animal populations. Ecol Lett 22: 1620-1680

555 Mousseau TA, Roff DA (1987) Natural selection and the heritability of fitness components.

$556 \quad$ Heredity 59: 181-197

557 Munday PL, Buston PM, Warner RR (2006) Diversity and flexibility of sex-change strategies in 558 animals. Trends Ecol Evol 21: 89-95

559 Nakagawa S, Schielzeth H (2013) A general and simple method for obtaining $\mathrm{R}^{2}$ from

560 generalized linear mixed effects models. Methods Ecol Evol 4: 133-142

561 Nijhout HF (2003) Development and evolution of adaptive polyphenisms. Evol Dev 5: 9-18

562 Petranka JW (1998) Salamanders of the United States and Canada. Smithsonian Institution Press, 563 Washington, D.C.

564 Pfennig DW (1990) The adaptive significance of an environmentally cued developmental switch 565 in an anuran tadpole. Oecologia 85: 101-107

566 Pfennig KS, Pfennig DW (2009) Character displacement: ecological and reproductive responses

567 to common evolutionary problem. Q Rev Biol 84: 253-276 
568 Pfennig DW, Wund MA, Snell-Rood EC, Cruickshank T, Schlichting CD, Moczek AP (2010)

569 Phenotypic plasticity's impacts on diversification and speciation. Trends Ecol Evol 25: 459-

$570 \quad 467$

571 Phillis CC, Moore JW, Buoro M, Hayes S, Garza JC, Pearse DE (2016) Shifting thresholds:

572 Rapid evolution of migratory life histories in steelhead/rainbow trout, Oncorhynchus mykiss.

$573 \quad$ J Hered 107: 51-60

574 R Development Core Team (2017) R: A language and environment for statistical computing. R

$575 \quad$ Foundation for Statistical Computing, Vienna, Austria.

576 Rowe L, Ludwig D (1991) Size and timing of metamorphosis in complex life cycles: time

577 constraints and variation. Ecology 72: 413-427

578 Ryan TJ, Plague GR (2004) Hatching asynchrony, survival, and the fitness of alternative adult

579 morphs in Ambystoma talpoideum. Oecologia 140: 46-51

580 Schielzeth H (2010) Simple means to improve the interpretability of regression coefficients.

$581 \quad$ Methods Ecol Ecol 1: 103-113

582 Scott DE, Komoroski MJ, Croshaw DA, Dixon PM (2013) Terrestrial distribution of pond-

583 breeding salamanders around an isolated wetland. Ecology 94: 2537-2546

584 Searcy CA, Gray LN, Trenham PC, Shaffer HB (2014) Delayed life history effects, multilevel 585 selection, and evolutionary trade-offs in the California tiger salamander. Ecology 95: 68-77

586 Semlitsch RD (1987) Paedomorphosis in Ambystoma talpoideum: Effects of density, food, and $587 \quad$ pond drying. Ecology 68: 994-1002

588 Semlitsch RD, Boone MD (2009) Aquatic mesocosms. In: Dodd CK (ed) Amphibian ecology 589 and conservation: a handbook of techniques. Oxford University Press, Oxford, pp 87-104 
590 Semlitsch RD, Harris RN, Wilbur HM (1990) Paedomorphosis in Ambystoma talpoideum:

591 maintenance of population variation and alternative life-history pathways. Evolution 44:

$592 \quad 1604-1613$

593 Semlitsch RD, Scott DE, Pechmann JHK (1988) Time and size at metamorphosis related to adult 594 fitness in Ambystoma talpoideum. Ecology 69: 184-192

595 Smith TB, Skúlason S (1996) Evolutionary significance of resource polymorphisms in fishes, 596 amphibians, and birds. Annu Rev Ecol Syst 27: 111-133

597 Sultan SE, Spencer HG (2002) Metapopulation structure favors plasticity over local adaptation. 598 Am Nat 160: 271-283

599 Tachiki Y, Koizumi I (2016) Absolute and relative assessments of individual status in status600 dependent strategies in stochastic environments. Am Nat 188: 113-123

601 Tomkins JL, Brown GS (2004) Population density drives the local evolution of a threshold 602 dimorphism. Nature 431: 1099-1103

603 Tomkins JL, LeBas NR, Unrug J, Radwan J (2004) Testing the status dependent ESS model:

604 population variation in fighter expression in the mite Sancassania berlesei. J Evol Biol 17:

$605 \quad 1377-1388$

606 Tomkins JL, Hazel WN (2007) The status of the conditional evolutionary stable strategy. Trends $607 \quad$ Ecol Evol 22: 522-528

608 Tomkins JL, Hazel WN, Penrose MA, Radwan JW, LeBas NR (2011) Habitat complexity drives 609 experimental evolution of a conditionally expressed secondary sexual trait. Curr Biol 21: 569$610 \quad 573$

611 Tomkins JL, Moczek AP (2009) Patterns of threshold evolution in polyphenic insects under 612 different developmental models. Evolution 63: 459-468 
613 Van Buskirk J, Cereghetti E, Hess JS (2017) Is bigger really better? Relative and absolute body

614 size influence individual growth rate under competition. Ecol Evol 7: 3745-3750

615 Voss SR, Kump DK, Walker JA, Shaffer HB, Voss GJ (2012) Thyroid hormone responsive QTL

616 and the evolution of paedomorphic salamanders. Heredity 109: 293-298

617 Warner RR, Fitch DL, Standish JD (1996) Social control of sex change in the shelf limpet,

618 Crepidula norrisiarum: size-specific responses to local group composition. J Exp Mar Biol

619 Ecol 204: 155-167

620 Warner DA, Shine R (2008) The adaptive significance of temperature-dependent sex

621 determination in a reptile. Nature 451: 566-568

622 Watkins TB, McPeek MA (2006) Growth and predation risk in green frog tadpoles (Rana

623 clamitans): a quantitative genetic analysis. Copeia 2006: 478-488

624 Werner EE (1986) Amphibian metamorphosis: growth rate, predation risk, and the optimal size 625 at transformation. Am Nat 128: 319-341

626 West-Eberhard MJ (2003) Developmental plasticity and evolution. Oxford University Press, 627 Oxford, UK.

628 Wheeler DE (1991) The developmental basis of worker caste polymorphism in ants. Am Nat $629 \quad 138: 1218-1238$

630 Whiteman HH (1994) Evolution of facultative paedomorphosis in salamanders. Q Rev Biol 69: $631 \quad 205-221$

632 Whiteman HH (1997) Maintenance of polymorphism promoted by sex-specific fitness payoffs. 633 Evolution 51: 2039-2044. 
634 Whiteman HH, Krenz JD, Semlitsch RD (2006) Intermorph breeding and the potential for

635 reproductive isolation in polymorphic mole salamanders (Ambystoma talpoideum). Behav

636 Ecol Sociobiol 60: 52-61

637 Whiteman HH, Sheen JP, Johnson EB, VanDeusen A, Cargille R, Sacco TW (2003)

638 Heterospecific prey and trophic polyphenism in larval tiger salamanders. 2003: 56-67

639 Whiteman HH, Wissinger SA, Denoël M, Mecklin CJ, Gerlanc NM, Gutrich JJ (2012) Larval

640 growth in polyphenic salamanders: making the best of a bad lot. Oecologia 168: 109-118

641 Wilbur HM (1997) Experimental ecology of food webs: complex systems in temporary ponds.

$642 \quad$ Ecology 78: 2279-2302

643 Wilbur HM, Collins JP (1973) Ecological aspects of amphibian metamorphosis. Science 182:

$644 \quad 1305-1314$

645 Wissinger SA, Whiteman HH, Denoël M, Mumford ML, Aubee CB (2010) Consumptive and

646 nonconsumptive effects of cannibalism in fluctuating age-structured populations. Ecology 91:

$647 \quad 549-559$

648 Ziemba RE, Collins JP (1999) Development of size structure in tiger salamanders: the role for

649 intraspecific intereference. Oecologia 120: 524-529

650 


\section{TABLES}

652 Table 1. Results from a multi-model inference approach that explicitly compared two sets of

653 candidate models of morph expression. All models included the stated fixed effects as well as a

654 random intercept that accounted for non-independence of individuals from the same tank.

655 Pseudo-R2 statistics were estimated using the approach advocated by Nakagawa \& Schielzeth

656 (2013). Although all analyses were conducted using the same responses from the same set of

657 individuals, convergence issues prevented us from fitting a single global model with all the of the

658 terms simultaneously. We therefore present results from approaches beginning with two separate

659 "full" models, even though some terms are shared between the two sets. 
661 Table 1.

\begin{tabular}{|c|c|c|c|c|c|c|c|c|}
\hline Approach & Rank & Fixed Effects & $\mathrm{k}$ & $\log$ Lik & $\mathrm{AIC}_{\mathrm{c}}$ & $\Delta$ & Weight & Pseudo-R ${ }^{2}$ \\
\hline \multirow{5}{*}{$\begin{array}{c}\text { Relative } \\
\text { Size (RS) } \\
\text { vs } \\
\text { SVL }^{1}\end{array}$} & 1 & RS & 3 & -48.13 & 102.5 & 0.0 & 0.510 & 0.468 \\
\hline & 2 & $\mathrm{RS}+\mathrm{SVL}$ & 4 & -47.44 & 103.3 & 0.8 & 0.343 & 0.462 \\
\hline & 3 & $\mathrm{RS}+\mathrm{SVL}+\mathrm{RS}: \mathrm{SVL}$ & 5 & -47.39 & 105.5 & 3.0 & 0.118 & 0.473 \\
\hline & 4 & SVL & 3 & -51.00 & 108.3 & 5.8 & 0.029 & 0.387 \\
\hline & 5 & Int. & 2 & -56.41 & 117.0 & 14.5 & 0.000 & 0.130 \\
\hline \multirow{5}{*}{$\begin{array}{c}\text { Relative } \\
\text { Size (RS) } \\
\text { vs } \\
\text { Density }\end{array}$} & 1 & RS & 3 & -48.13 & 102.5 & 0.0 & 0.863 & 0.468 \\
\hline & 2 & RS + Density & 5 & -47.96 & 106.6 & 4.1 & 0.112 & 0.464 \\
\hline & 3 & RS + SVL + RS:Density & 7 & -47.17 & 107.7 & 7.2 & 0.024 & 0.497 \\
\hline & 4 & Int. & 2 & -56.41 & 117.0 & 14.5 & 0.001 & 0.130 \\
\hline & 5 & Density & 4 & -56.41 & 121.3 & 18.8 & 0.000 & 0.129 \\
\hline
\end{tabular}

662

663 1- As described in the main text, SVL was centered to improve model convergence. 

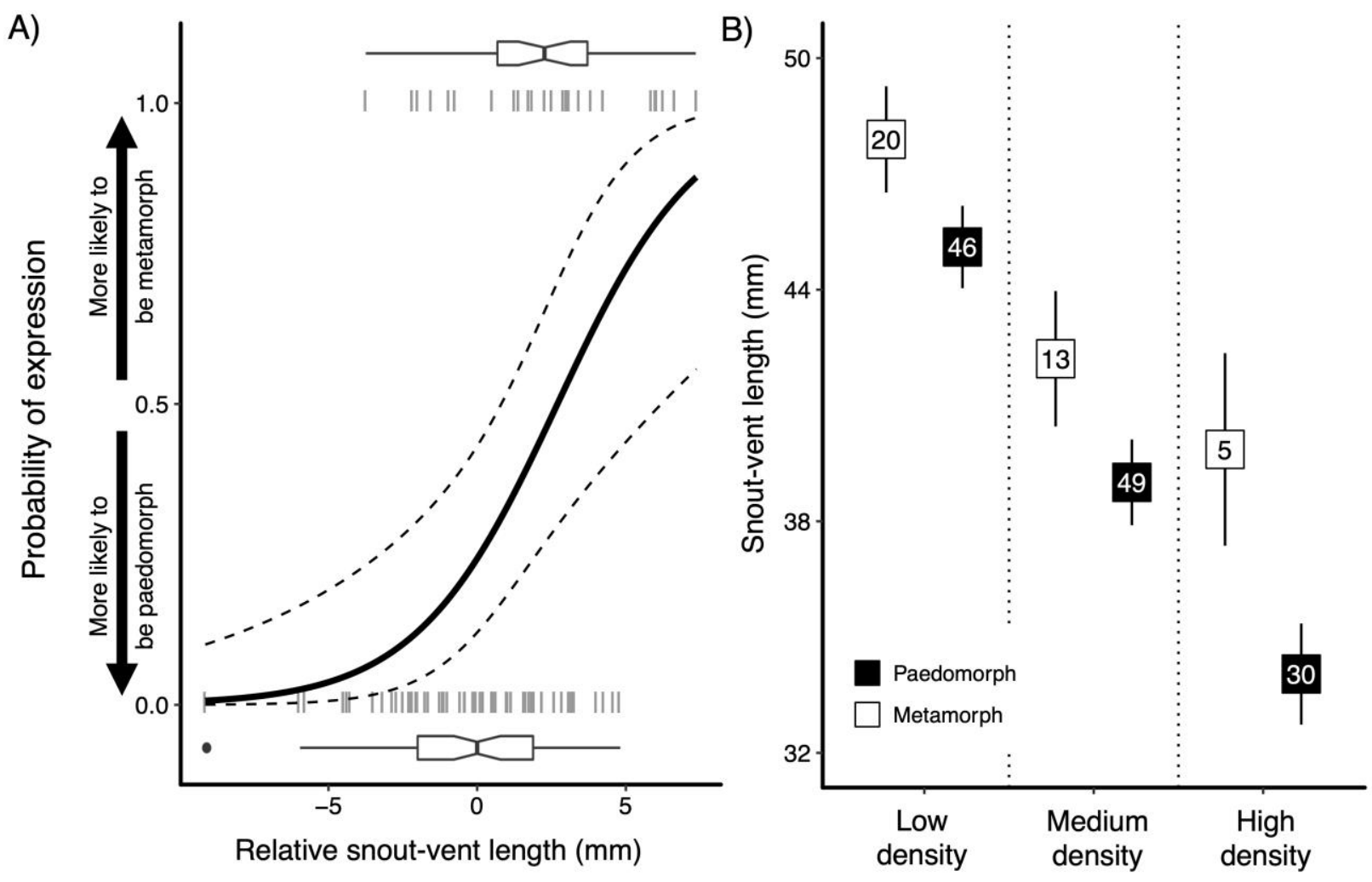

666

667 Figure 1. The effects of relative size on morph expression. Metamorphic A. talpoideum tended to 668 be (A) the relatively largest individuals within a tank and (B) the largest individuals within a

669 density treatment. A) Each tick mark represents an individual that either became a metamorph

670 (1) or a paedomorph (0), and box plots are provided to aid visualization of the relative-size

671 distributions. The line is fitted from the best-supported mixed-effects model reported in the main

672 text, and it illustrates the predicted probability of an individual of a given relative size within a

673 tank becoming either a paedomorph or a metamorph ( $\pm 95 \%$ CIs $)$. B) Squares represent the least-

674 squares-mean SVL ( $\pm 95 \%$ CIs) for individuals expressing that morph. Numbers inside points

675 are the sample sizes. 